\title{
Self-Organizing Wireless Monitoring System for Containers
}

\author{
Ryszard J. Katulski, Jacek Stefański, Jarosław Sadowski, \\ Sławomir J. Ambroziak, and Bożena Miszewska \\ Department of Radiocommunication Systems and Networks, \\ Gdansk University of Technology \\ 11/12 Gabriela Narutowicza Street \\ 80-952 Gdańsk \\ rjkateeti.pg.gda.pl, jstef@eti.pg.gda.pl, \\ Jaroslaw.Sadowski@eti.pg.gda.pl, sj_ambroziak@eti.pg.gda.pl, \\ bozena.miszewska@yahoo.pl
}

\begin{abstract}
This paper presents a description of new global monitoring system for containers, with its layer-modular structure, as a solution for enhance security and efficiency of container transport with particular emphasis on the practical implementation of that system for maritime container terminals. Especially the Smart Container Module (SCM) architecture and its operation as a part of the Self-Organizing Container Monitoring Network is presented.
\end{abstract}

\section{Introduction}

The first ship with containers, SS Ideal X, sailed from Newark for Houston, on 26th April 1956 and opened a new age in transportation. Currently container transport includes more than $90 \%$ of the global trade and takes place along trade lanes, both marine and terrestrial. However, despite all the advantages of this mode of transport, it carries a lot of risk, as a result of the exploitation of containers. First of all, containers are closed during the transportation, so the load on their interior remains beyond any control. For this reason, in peer-reviewed literature on the safety of transport, container is known as the Trojan Horse of twenty-first century. In this state of affairs it is particularly important issue of controlling and monitoring of cargo container, both during transportation and storage.

This issue became a subject of research at the Department of Radiocommunication Systems and Networks in the Gdansk University of Technology, where the Group of Self-Organizing Ad-Hoc Wireless Sensor Networks was founded. This Group developed the original concept of a global wireless system for monitoring of containers cargo, with particular emphasis on the practical implementation of that system for maritime container terminals $[1,2]$.

\section{General Characteristics of the System}

\subsection{A Global Grasp of the System}

The proposed new monitoring system solution for containers has a layer-modular structure (Fig.1). The basic layer of the system is the Smart Container Module (SCM), 
designed for installation on containers and eventually should be an integral part of each container. Another, higher layer of the system is the Ship Subsystem - the wireless self-organize network, based on the 802.11 standard, working on the container ship. Due to the fact that the typical container ship can carry up to dozen thousand containers, there is a necessity for the location a special database on the ship. Data about all containers are regularly sent to the ship network controller. Information can also be sent on request.

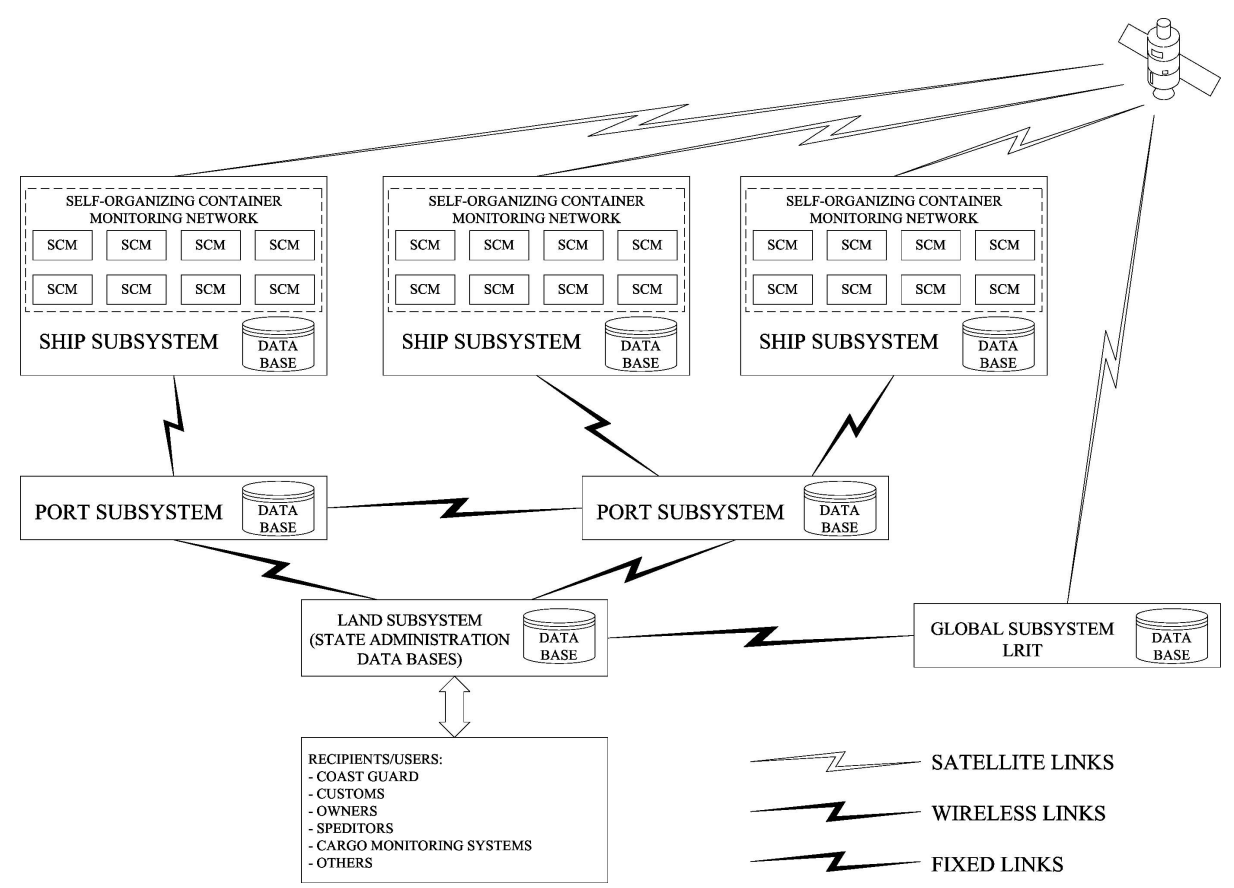

Fig. 1. Concept of the global wireless monitoring system for containers

Container terminals and sea ports form third layer of the system - the Port Subsystem with the Port Administration Data Base, equipped with wireless access points located at entrances to ports and wireless radio networks covering container depot. All of the Port Subsystems located inside the country are connected to each other and provide all data to the Land Subsystem - The State Administration Data Base. The Land Subsystem is the fourth layer of the monitoring system.

Transmission of information between each part of the system is in accordance with certain rules, using an appropriate transmission medium. In the case of a container ship, the period of reporting depends on its position, i.e. on distance to the mainland, the home port and/or destination port. Frequency of sending the relevant data increases with the approach to the mainland.

The Global Subsystem is the highest layer of the monitoring system, which can use the database of a new LRIT (Long Range Identification and Tracking) system for longrange ship monitoring. Data are transferred to the global database via satellite, when the vessel is on the open sea. Approaching the mainland and entering the harbour, 
information are communicated to the Port Subsystem that supports appropriate area. Transmission of this information is carried on using the GPRS system. It is also necessary to realize co-operation and data exchange between local and global databases, and also co-operation between databases inside the country. It can be realized by means of fixed lines, e.g. optical fiber.

The recipients (users) form a separate layer of the system. We can distinguish two classes of users: the services responsible for cargo security and the carriers. The first group is formed by the relevant services, such as Coast Guard, Customs and Police. The owners and the shipping companies belong to second group of users, which access to the system is carried out by authorized employees.

\subsection{The Smart Container Module}

The Smart Container Module is one of the most important elements of the monitoring system [1]. Figure 2 depicts its functional diagram.

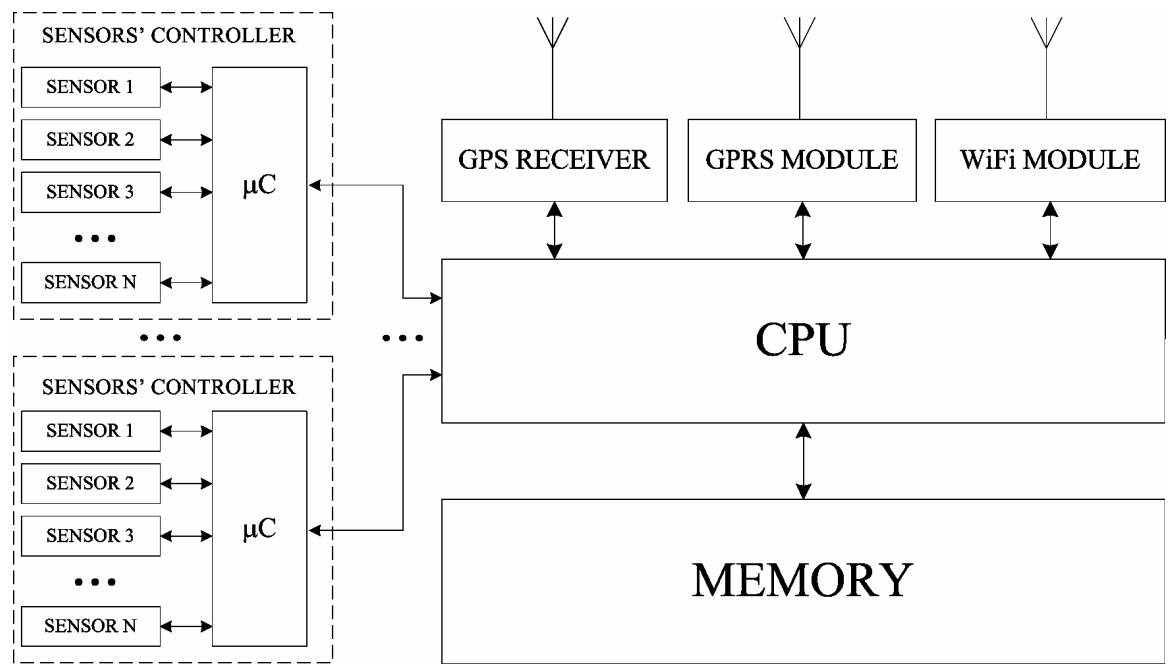

Fig. 2. Functional diagram of the Smart Container Module

The versatility of the SCM stems from the possibility of parameters measuring and monitoring of state within the container. To this end, the module is equipped with various sensors placed inside the container [3]. This sensor network enables detection of movement, temperature changes, pressure, humidity, presence of gases or radioactive substances, etc. An important aspect is also the location of the container, so each SCM is equipped with the GPS receiver. The GPRS module and the WiFi module are components of SCM. This parts of container module enable communication with port database (GPRS) or the local wireless network working on the container ship (WiFi).

Transportation documents concerns shipment (such as contents of the container, a shipper, a point of shipment, a point of destination and other information) are stored in SCM's memory. This data are sent to the appropriate database, whenever necessary. 
In case of lack of connection with the radio resources of the system, it is necessary to store data about status of container in the module's internal memory and send them shortly after the SCM gets in a range of network.

The container module is characterized by very low power consumption and thus it enables unattended work for a long time (from several months to even few years). Equally important is compact construction of the SCM and to minimize its dimensions. The module's casing is matched to construction of the container, to damage prevent during transshipment. It is resistant to the weather conditions and it also has got a protection against removal or modification of its interior, with automatic alert generating in these cases.

During the research on the SCM, it was decided to use two types of industrial computers: the Axiomtek SBC84710 and the Advantech ARK1380. Three prototypes of the SCM have been produced basing on these computers. The Smart Container Module prototype using the ARK1380 is shown in Figure 3.

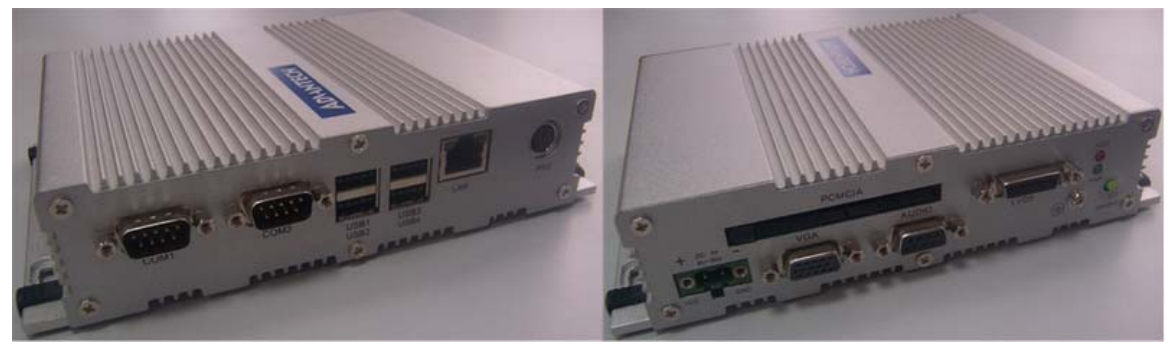

Fig. 3. A prototype of the Smart Container Module

The module is enclosed in a sealed casing and there are various connectors available, as following:

- $C O M 1, C O M 2$ - used to connect Sensors' Controllers in various configurations,

- USB1, USB2, USB3, USB4 - two of them are used to connect external GPRS and GPS modules, next two are designed for future purposes,

- $L A N$ - used to connect the SCM with the ship/port local network, in case of usage the SCM as a gateway,

- $D C I N$ - used to connect the supply voltage range from $9 \mathrm{~V}$ to $35 \mathrm{~V}$, but because of the usage of this voltage by Sensors' Controllers, it is recommended not to exceed a value of $15 \mathrm{~V}$,

- PCMCIA - used to connect the WiFi module,

- $V G A$ - used to connect the monitor,

- $P S 2$ - used to connect the keyboard,

- $A U D I O$ - not used, designed for future purposes,

- $L V D S$ - not used, designed for future purposes.

Additionally, there are LEDs placed on the SCM's casing, which indicate supply voltage connection and work of the hard disk. There is also the on/off button placed on the casing. 
The internal construction of the Smart Container Module is presented on an example of the SCM prototype based on SBC84710. The top view of internal construction of the SCM, with marked available interfaces, connectors, power supply voltage and connected the WiFi module is shown in Figure 4.

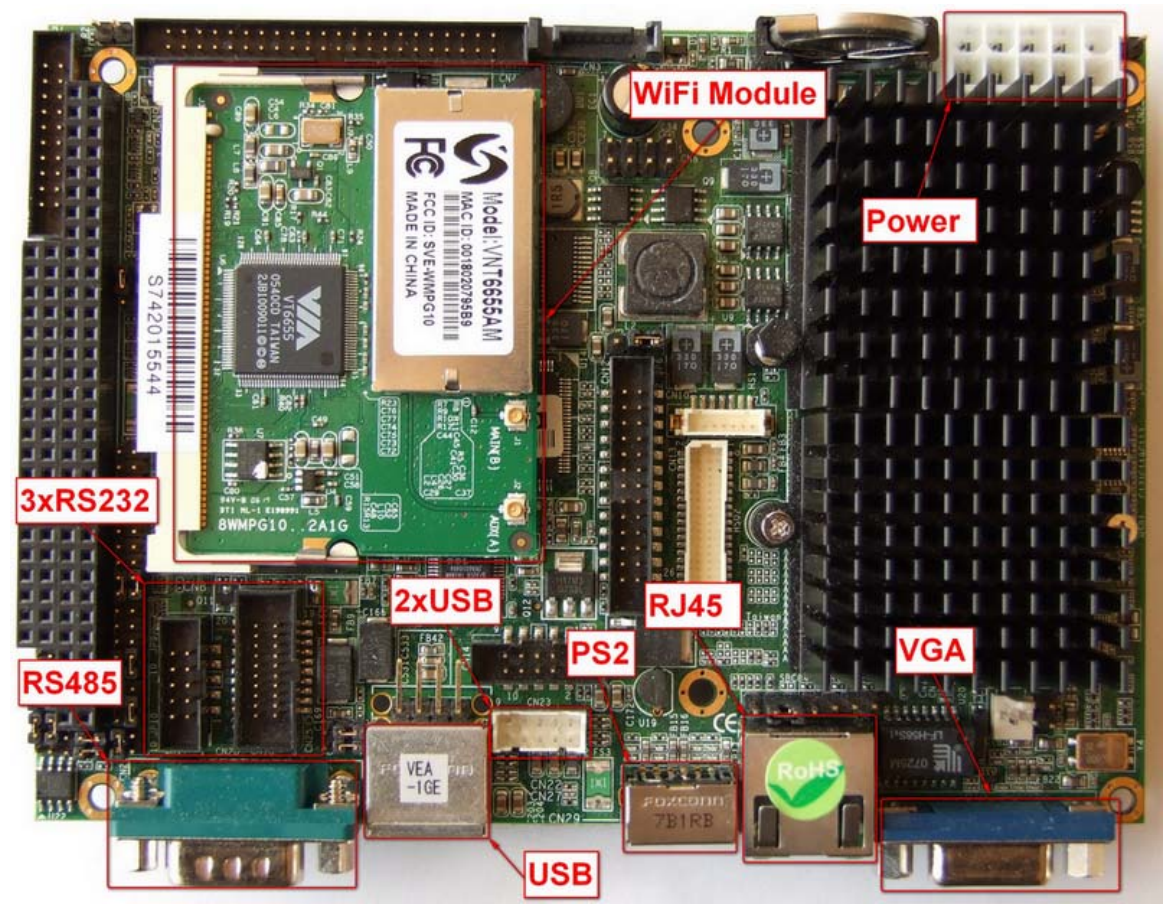

Fig. 4. Top view of the internal SCM construction

The bottom view of the internal SCM construction, with marked the RAM chip and the Compact Flash Card, which stores the operating system and all of the SCM's data is shown in Figure 5.

The container module is powered by two batteries thus it enables unattended work for a long time, which depends on batteries capacity. The first of batteries has a smaller capacity and it is enclosed in the SCM's casing. The second of batteries could have as large capacity as necessary. It is placed inside the container and connected to SCM. The SCM is equipped with a voltage control circuit, whereby it is possible to distinguish SCM turning off for lack of power against other cases.

The Sensors' Controller have been designed in a manner making construction of the measuring module independent from a type of using computer model and to enable free deployment of reconfiguring sensors inside the container. The exemplary Sensors' Controller board is shown in Figure 6.

Composition of this circuit includes three parts: the microcontroller, the RS485 converter with auxiliary circuits and the set of sensors dedicated to the specific cargo container. Sensors, which can be connected the Sensors' Controller with: 
- sabotage - this sensor allows to detect a sabotage, e.g. SCM's casing tamper or cutting off any of Sensors' Controllers,

- door switch - this sensor indicates whether or not the door of the container is open or closed,

- motion - this sensor allows to detect movement inside the container,

- smoke - this sensor allows to detect smoke inside the container,

- temperature - this sensor measures ambient temperature inside the cargo container,

- humidity - this sensor measures relative humidity inside the cargo container,

- acceleration - this sensor measures acceleration on three axes (range to $10 \mathrm{~g}$ or to $50 \mathrm{~g})$.

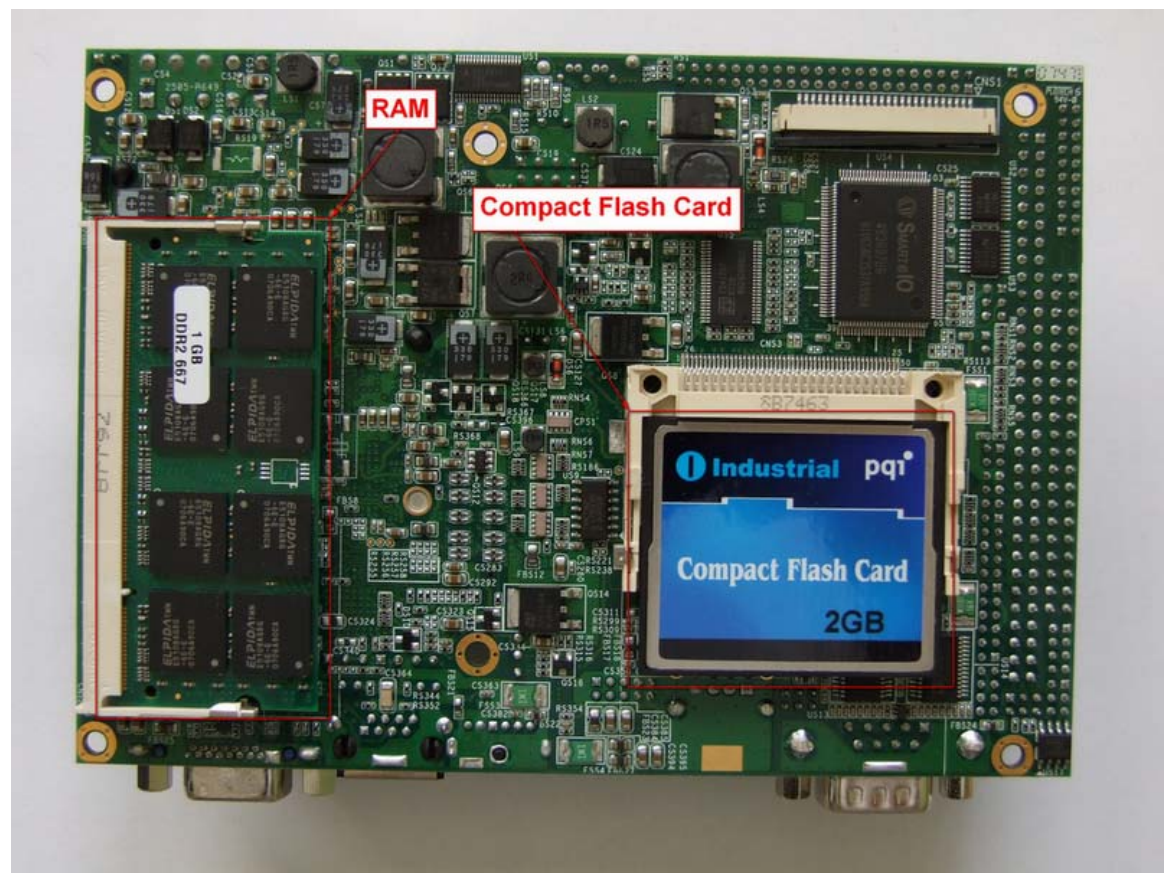

Fig. 5. Bottom view of the internal SCM construction

Usage of Sensors' Controllers allows connect many sensors to the SCM simultaneously. Additionally it allows to unrestricted changes types of sensors and their deployment inside the container.

\section{Self-Organizing Network of SCM Modules}

Because of the vertical way of containers storage on container ships, there is a problem of access to the on-board wireless network for Smart Container Modules mounted on the containers at the lowest level. Additionally, GPS satellites are beyond the sight of view of the GPS receiver, installed in the adversely located SCM modules, thus it is impossible to read the geographical position. In order to solve this problem SCM 
modules have been programmed in a manner that allows them to self-organize in adhoc network. During the research a new algorithm for data transmission control over a multi-hop ad-hoc network (assuming slow-moving nodes) has been developed.

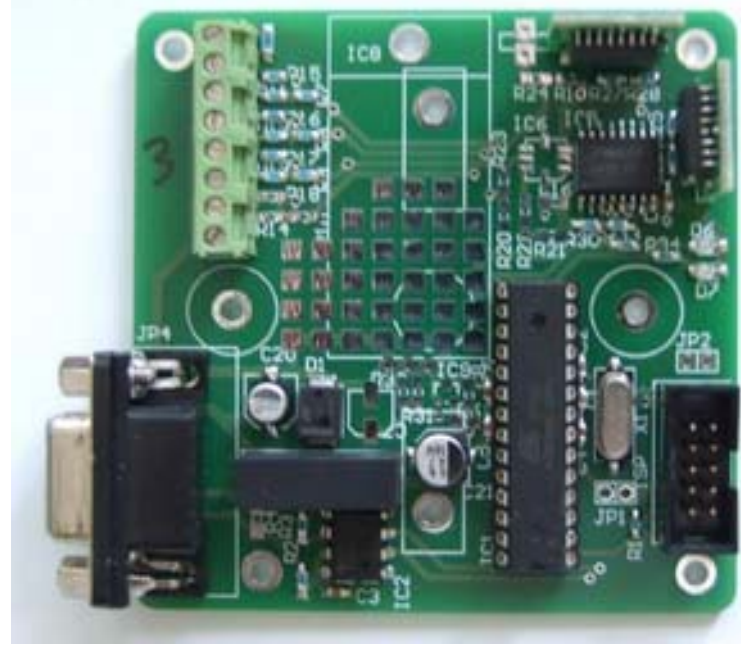

Fig. 6. The sensors' controller board

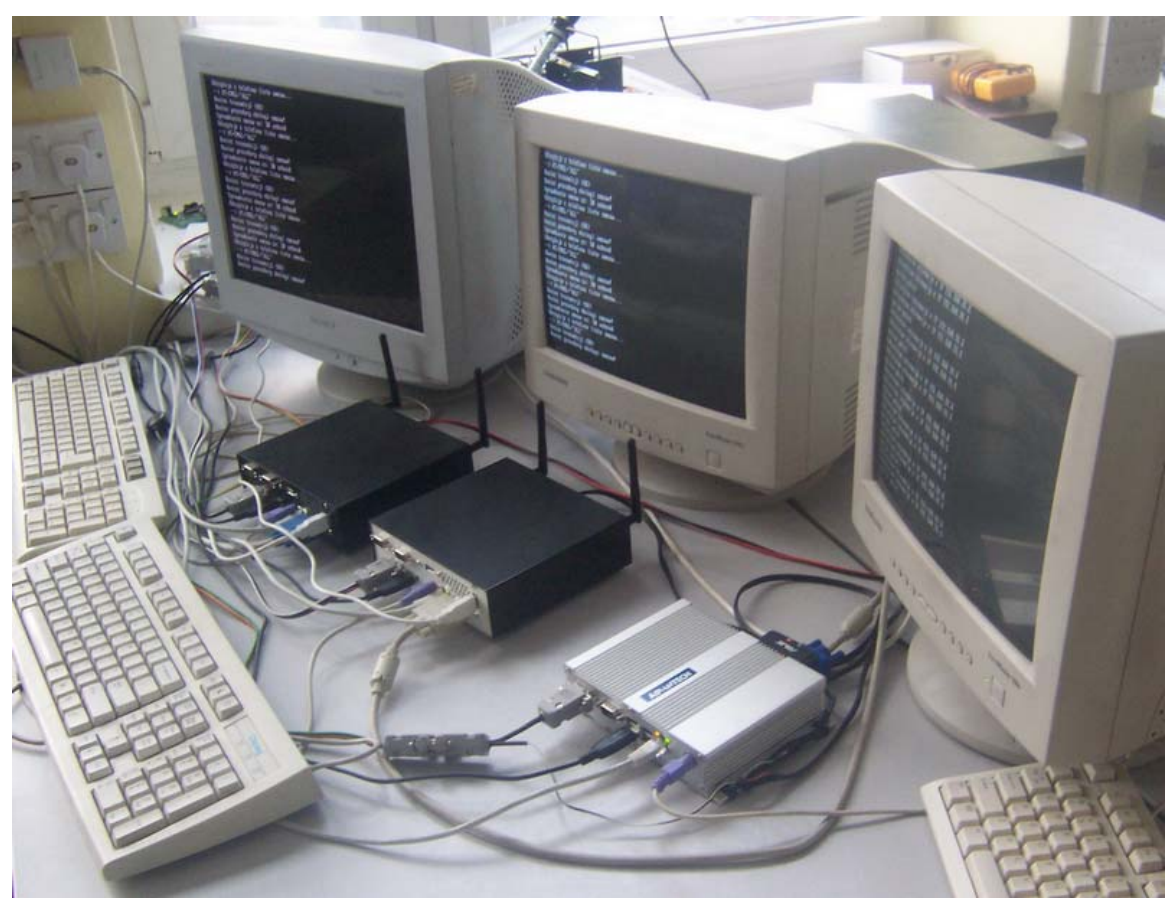

Fig. 7. The SCF self-organizing ad-hoc network tests 
By dint of this solution it is possible to transfer data about cargo to the Ship Subsystem's database, even from the Smart Container Module installed on the lowest container. The data about the cargo are transferred to adjacent modules by the WiFi radio link. If module has a connection to the on-board wireless network, it will forward data to the database. If this module is beyond the reach of the on-board wireless network, it will forward the data to the next SCM, etc. Additionally, the self-organizing ad-hoc network enables SCM modules beyond the reach of GPS system to read and save the geographical position on the basis of data from modules at the top of containers' stack. The ability to self-organize modules in the ad-hoc network can also be useful in harbours or container terminals, where direct access to the Port Subsystem's wireless network or receiving signals from GPS satellites can be impossible. Figure 7 depicts the laboratory for testing the Self-Organizing Container Monitoring Network composed of three Smart Container Modules.

The results of these tests have proved the ability of testing network to self-organize in real time, especially to choose SCM module to communicate with the database.

\section{User Interface}

In the course of the research, the user interface including a database storing all data about the system has been created. Depending on the granted access privileges, user may get the following information: state of the system, defined types of containers, registered containers (with the assigned sensor network), ongoing and completed shipments and communication history. In addition, there is the ability to users manage (adding/removing user, changing password) only for users with administrator privileges.

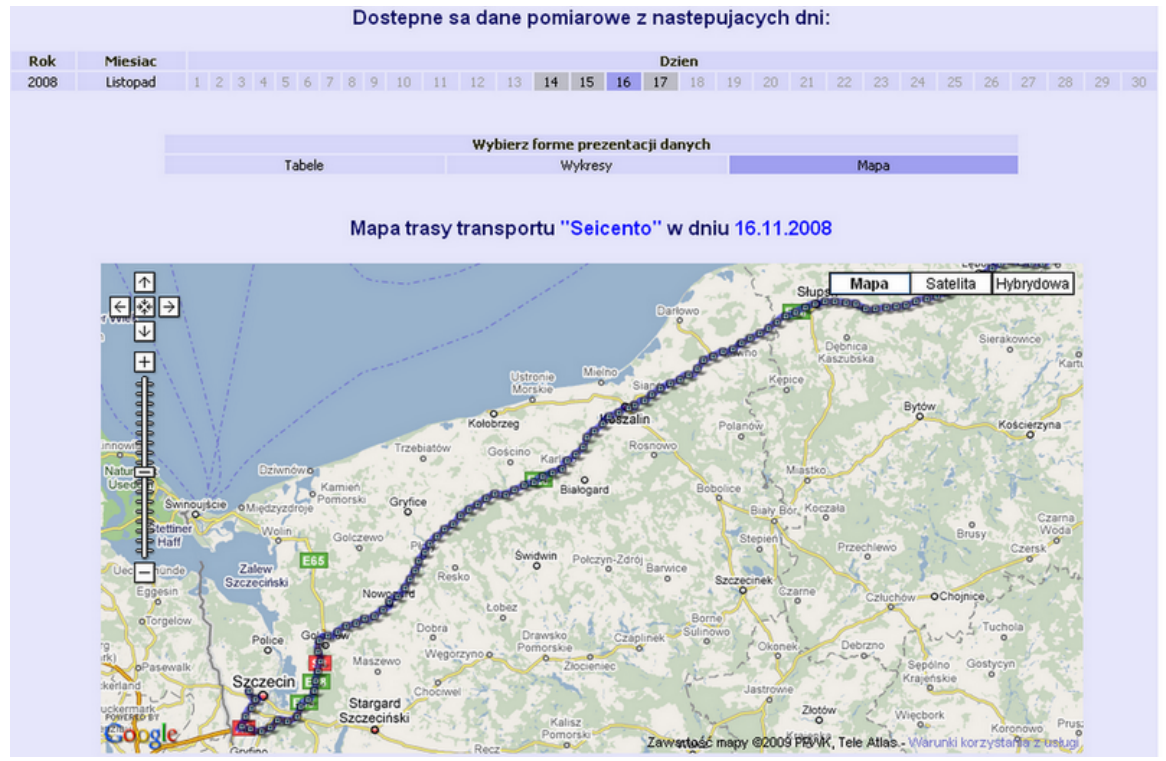

Fig. 8. Sample view of the user interface with a map of the transport route 
Each transport can be remotely monitored. Cargo information (parameters from the sensor network, current position and route of transport) are available in three forms: a table, graphs and a map. Figure 8 depicts a map of portion of the transport route. This function uses the Google Maps available for free by Google.

Data can also be presented in a transparent form of graphs, which are created on the basis of data from different sensors. Sample record of the temperature inside the cargo is presented in Figure 9.

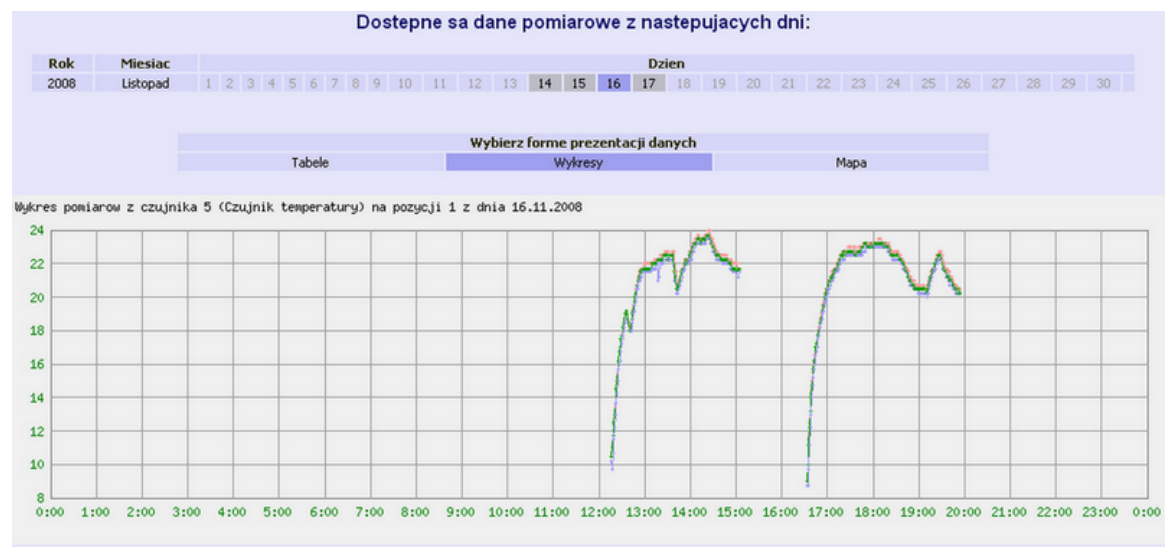

Fig. 9. Sample view of the user interface in the control of temperature scope

Using the interface is intuitive and user-friendly, which greatly facilitates access to the system at any point on earth with access to the Internet.

\section{Conclusion}

This concept of global system for monitoring of cargo, especially self-organizing monitoring network consisting SCM modules is a part of a wider security subject matter, which currently has a high priority, both in Poland and the world. In addition, increasing the efficiency of container transport is possible by dint of the safe and efficient user interface.

Acknowledgments. This new solution of system for wireless monitoring of containers is funded by the Polish Ministry of Science and Higher Education, as a part of research and development project No R02 012 01. The authors express their sincere thanks for allocated funds for this purpose.

\section{References}

[1] Katulski, R., Niski, R., Stefański, J., Żurek, J.: Concept of the container monitoring system in Polish harbours. In: 2006 IEEE Conference on Technologies for Homeland Security, Enhancing Transportation Security and Efficiency, Boston, USA, June 7 (2006)

[2] Katulski, R.J., Ambroziak, S.J., Sadowski, J., Stefański, J.: Containers wireless monitoring systems, Przegląd Telekomunikacyjny, No 12/2008, p. 1065 (2008)

[3] Karl, H., Willig, A.: Protocols and Architecture for Wireless Sensor Networks. Wiley \& Sons, Chichester (2006) 